\title{
Calibration of the length of a chain of single gold atoms
}

\author{
C. Untiedt, ${ }^{1}$ A. I. Yanson, ${ }^{1}$ R. Grande, ${ }^{2}$ G. Rubio-Bollinger, ${ }^{2}$ N. Agrait ${ }^{2}$ S. Vieira ${ }^{2}$ and J.M. van Ruitenbeek ${ }^{1}$ \\ ${ }^{1}$ Kamerlingh Onnes Laboratorium, Leiden University, PO Box 9504, NL-2300 RA Leiden, The Netherlands \\ ${ }^{2}$ Laboratorio de Bajas Temperaturas, Departamento de Física de la Materia Condensada C-III, Universidad Autónoma de Madrid, \\ E-28049 Madrid, Spain
}

(Received 20 February 2002; revised manuscript received 16 May 2002; published 29 August 2002)

\begin{abstract}
Using a scanning tunnelling microscope or mechanically controllable break junction it has been shown that it is possible to control the formation of a wire made of single gold atoms. In these experiments an interatomic distance between atoms in the chain of $\sim 3.6 \AA$ was reported which is not consistent with recent theoretical calculations. Here, using precise calibration procedures for both techniques, we measure the length of the atomic chains. Based on the distance between the peaks observed in the chain length histogram we find the mean value of the interatomic distance before chain rupture to be $2.5 \pm 0.2 \AA$. This value agrees with the theoretical calculations for the bond length. The discrepancy with the previous experimental measurements was due to the presence of He gas, that was used to promote the thermal contact, and which affects the value of the work function that is commonly used to calibrate distances in scanning tunnelling microscopy and mechanically controllable break junctions at low temperatures.
\end{abstract}

DOI: 10.1103/PhysRevB.66.085418

PACS number(s): 62.25.+g, 73.40.Jn, 73.63.Rt

\section{INTRODUCTION}

In the last few years, there has been a significant advance in the understanding of the electronic properties of atomicsized contacts. This has been possible thanks to the use of two techniques: scanning tunnelling microscopy (STM) (Refs. 1 and 2) and the mechanically controllable break junction (MCBJ). ${ }^{3}$ In both cases the distance between two electrodes is controlled by means of a piezoelectric transducer which allows for relative displacements of the electrodes down to a resolution in the range of picometers.

In these experiments the current that traverses the contact between two electrodes, at a given bias voltage, is measured as a function of the relative displacement of these electrodes. As the contact is broken, the current changes smoothly during elastic elongation stages, decreasing suddenly in plastic deformations stages. ${ }^{4,5}$ In the last stage before breaking the contact, just a few atoms determine the electronic transport and the conductance is given by the Landauer formula

$$
G=\frac{2 e^{2}}{h} \sum_{n=1}^{N} T_{n}
$$

Here, $N$ is the number of available channels for the electrons traversing the contact, $e$ is the electron charge, $h$ is Planck's constant, and $T_{n}$ is the transmission probability of the $n$th channel. Just before the contact is broken, when there is just a single atom at the contact, the conductance for monovalent metals, such as gold, has been shown ${ }^{6}$ to be due to a single conductance channel with transmission probability close to unity and therefore with a conductance close to the value $2 e^{2} / h$.

It was observed that occasionally the conductance of the one-atom contact for gold remains constant while the distance between the two electrodes increases by more than an interatomic distance, as it is shown in Fig. 1. When it finally breaks, in order to make contact again it is necessary to decrease the interelectrode distance by the same distance.
From such observations it was concluded that in these cases a wire only one atom thick was formed between the two electrodes. ${ }^{7}$ These wires have also been observed by transmission electron microscopy (TEM). ${ }^{8,9}$ The Au-Au bond length was reported to be $3.6 \AA( \pm 30 \%)$ and $3.6-4.0 \AA$ in Refs. 7 and 8, respectively. It was recently shown that, apart from $\mathrm{Au}$, chain formation can be observed in Pt and Ir. ${ }^{10,11} \mathrm{In}$ this paper we concentrate on Au contacts.

Several calculations have confirmed the possibility of the formation of an atomic chain of gold atoms when stretching the monatomic contact. ${ }^{12-16}$. However, in all cases a large discrepancy with the reported experimental bond length $(\sim 3.6 \AA)$ was found. The calculations use different methods including $a b$ initio calculations using the local-density approximation ${ }^{13-15,17,18}$ and molecular-dynamics simulations using effective-medium theory ${ }^{12}$ or tight-binding approximations. ${ }^{16}$ For the wire's equilibrium bond length the

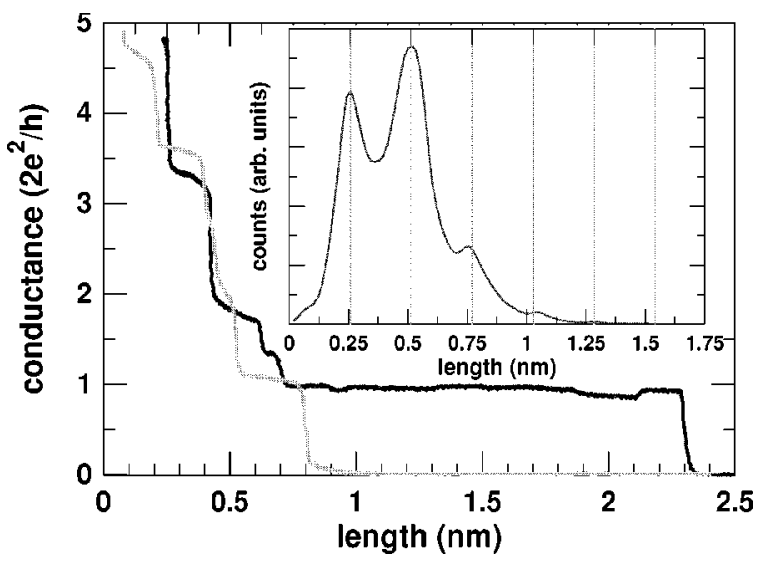

FIG. 1. Evolution of the conductance for a gold contact as a function of the interelectrode distance recorded with the MCBJ technique. The last plateau of the conductance corresponds to an atomic chain of about six atoms long. The inset shows a histogram made from 10000 recordings of the last plateau length obtained from individual conductance curves taken at $4.2 \mathrm{~K}$ in vacuum. 
different calculations give a distance between 2.32 and $2.55 \AA$, and an upper limit after stretching of $3.0 \AA$, much smaller than the one reported in the experiments.

In this paper we show how the interatomic distance in these atomic wires can be estimated from the conductance vs electrode displacement curves. Using this method we obtain for gold chains at low temperature $(4.2 \mathrm{~K})$ an interatomic distance of $2.5 \pm 0.2 \AA$ at the average maximum tensile stress at the moment of fracture.

\section{CALIBRATION METHODS}

Since the separation between the peaks in the length histogram can provide information on the bond distance in the chains it is crucial to have a good calibration of the displacement of the electrodes as a function of the voltage which is applied to the piezo element of the STM or MCBJ. The various methods that we have used to calibrate our STM and MCBJ are described below.

\section{A. Tunnel barrier}

The exponential dependence of the current on the vacuum gap can be used to make a rough calibration in STM, and until very recently ${ }^{20}$ it was the only way to obtain a calibration of the interelectrode displacements in the MCBJ. One makes use of the well-known dependence of the tunnel current $I_{T}$ between two electrodes which are separated by a distance $d$, when a small voltage $V_{0}$ smaller than the work function of the electrodes is applied, ${ }^{21}$

$$
I_{T}\left(V_{0}\right)=K V_{0} e^{-2 d \sqrt{2 m \phi / \hbar^{2}}},
$$

where $m$ is the mass of the electron, $\phi$ is the height of the tunnel barrier, approximately given by the mean value of the work function of the two electrodes, $K$ is a constant which is related to the area of the electrodes and to the electronic density of states at the Fermi level.

The exponential dependence of the tunnel current with the interelectrode distance makes it very easy to control that distance and this is the basis of operation of the STM. If we represent on a semilog scale the variation of the current as function of the voltage $V_{p}$ applied to the piezo element (see Fig. 2) for the slope $\gamma$ we obtain the following expression:

$$
\gamma=-\frac{\sqrt{2 m \phi}}{\hbar} \frac{2 \Delta d}{\Delta V_{P}} .
$$

This immediately gives us a calibration of the distance as

$$
\kappa=\frac{\Delta d}{\Delta V_{p}}=\frac{-\hbar \gamma}{2 \sqrt{2 m \phi}} .
$$

This expression is very simple and indeed in experiments with clean electrodes an exponential behavior of the current as a function of $V_{P}$ is found, which would make this a suitable method for calibration of the response of the system to the voltage applied to the piezoelectric transducer. Although a more realistic description for the tunnel barrier must include electron screening effects, it has been $\operatorname{argued}^{22}$ that

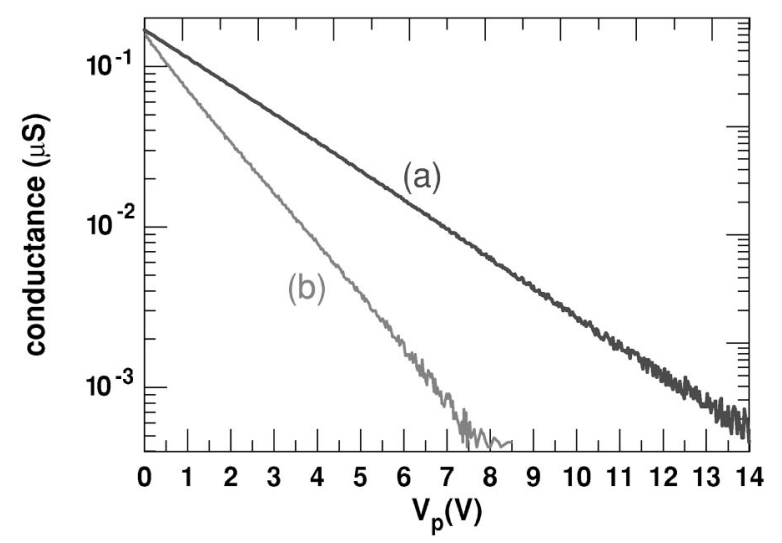

FIG. 2. Exponential dependence of the current as function of the piezo voltage at a fixed bias voltage $V=100 \mathrm{mV}$ when the two electrodes are separated by a tunnel barrier in vacuum (a) or in a He atmosphere (b).

these effects nearly cancel in the logarithmic derivative, at least for not too small distances.

A problem that arises when using this method is that the value of the tunnel barrier $\phi$ is dependent on the local work function of the closest parts of the two electrodes. This local work function depends mainly on the material with some variation due to surface distortion and crystal orientation [e.g., the work function for gold in the (100) direction is 5.47 $\mathrm{eV}$ while for the (111) direction it has a value of $5.31 \mathrm{eV}$ (Ref. 24)]. However, the largest deviation is due to the use of helium. Helium gas is commonly used to promote thermal contact for cooling of the STM or MCBJ. It was generally believed that helium gas does not significantly influence the electron tunneling between two metallic electrodes. However, very recently it has been found that atomic layers of adsorbed helium can affect dramatically the work function measured with this technique. ${ }^{23}$ Since the apparent work function was seen to increase for a He pressure of only 0.01 Torr by $80 \%$ above the clean surface value, errors of up to $34 \%$ are introduced in the distance calibration due to the presence of a helium atmosphere. For this reason, in the MCBJ experiments described below we avoid using helium as a thermal exchange gas.

\section{B. Gundlach oscillations}

A different method for calibrating the MCBJ, based on the Gundlach oscillations, has been developed by O.Yu. Kolesnychenko et al. ${ }^{20}$ The Gundlach oscillations, ${ }^{25}$ or fieldemission resonances, are observed in the tunnel conductance when a voltage higher than the work function of the electrodes is applied between them.

As illustrated in Fig. 3 when the applied voltage $V$ across the tunnel junction is larger than the work function of the electrodes, $\phi_{1,2}$, part of the barrier region becomes classically accessible. In this case the wave function of the electrons in the region between the electrodes will be determined by the superposition of the incoming and reflected waves at the interfaces. This mechanism will give rise to periodic maxima of the transmission as a function of bias voltage 


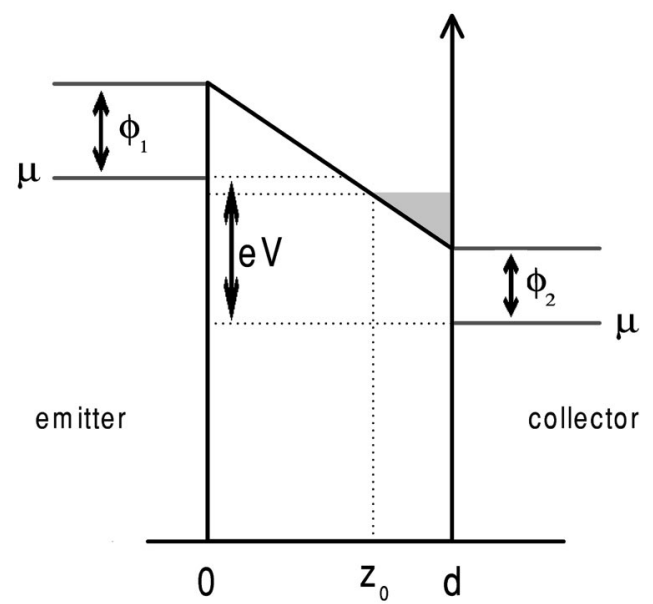

FIG. 3. Energy diagram for field-emission oscillations. Horizontal: $z$ coordinate parallel to the current direction. Vertical: energy. $\phi_{1}$ is the work function for the left electrode and $\phi_{2}$ that for the right electrode. The chemical potential for the two electrodes are shifted by the applied voltage $e V$.

when new resonant states are formed between the electrodes.

Using the model for this problem proposed by Gundlach, ${ }^{25}$ Kolesnychenko et al. obtained an expression for the differential conductance as a function of bias voltage given by

$$
\frac{d I(V)}{d V} \sim A(V) \cos \left[\zeta_{d}(V)\right] .
$$

The amplitude of the oscillations, $A(V)$, decreases with voltage as $V^{-3 / 2}$ and the argument for the cosine function is given by

$$
\zeta_{d}(V)=\frac{4}{3} \frac{\sqrt{2 m}}{\hbar} \frac{\left(e V-\phi_{2}\right)^{3 / 2}}{e F},
$$

where $F$ is the electric-field strength in the vacuum gap.

The relation between the peak position $V_{n}$ of the oscillations in Eq. (5) and their index can be found by equating Eq. (6) to $2 n \pi$ :

$$
e V_{n}=\phi_{2}+\left(\frac{3 \pi \hbar}{2 \sqrt{2 m}}\right)^{2 / 3} F^{2 / 3} n^{2 / 3} .
$$

During the experiment we keep $F$ constant by applying a feedback to the piezo voltage in order to maintain the current constant. From a plot of $V_{n}$ versus $n^{2 / 3}$ the work function $\phi$ is obtained as the intercept at the voltage axis and from the slope $\sigma$ of the curve we obtain the field strength

$$
F=\frac{2 \sqrt{2 m}}{3 \pi \hbar} \sigma^{3 / 2}
$$

The distance between the two electrodes will then be related to $F$ and the applied bias voltage according to

$$
d=\frac{1}{e F}(e V+\Delta \phi)
$$

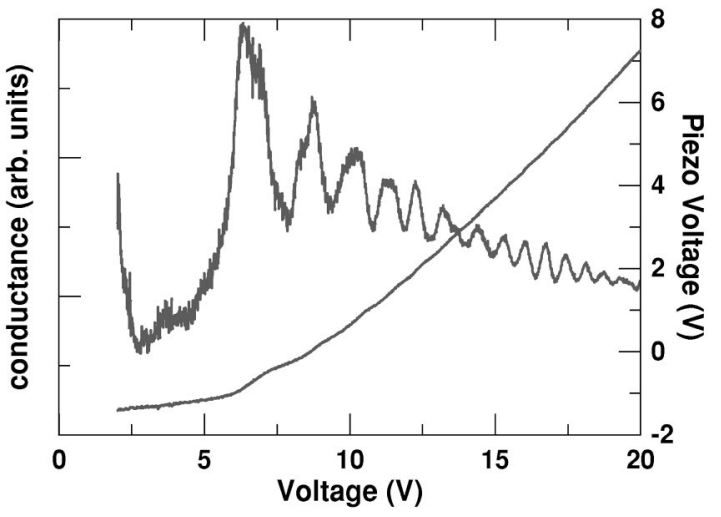

FIG. 4. Evolution of conductance and piezo voltage as the applied voltage across the tunnel junction is increased, while keeping the current constant. The analysis of this curve gives a value of 5.3 $\mathrm{eV}$ for the tunnel barrier indicating that the surface is free of adsorbates.

where $\Delta \phi$ is the difference in the work function between the two electrodes. Using these expressions the procedure to make the calibration using the Gundlach oscillations will be as follows: we record the evolution of the conductance, as well as the piezo voltage $V_{p}$, as a function of the applied bias voltage while keeping the current constant (see Fig. 4). Then using Eqs. (7) and (8) we can calculate the field strength $F \simeq 1.087 \sigma^{3 / 2}[\mathrm{~V} / \mathrm{nm}]$. Finally using Eq. (9) and the response of the feedback to the voltage changes applied to the junction we can obtain

$$
\kappa=\frac{\Delta d}{\Delta V_{p}}=\frac{1}{F} \frac{\Delta V}{\Delta V_{p}}
$$

for the response at high voltages, where the variation is approximately linear.

\section{Interferometric calibration}

The interferometric calibration is a very accurate method for distance calibration. We have used an all-fiber interferometer similar to those used in atomic force microscopy ${ }^{26}$ to calibrate our STM used in the experiments on atomic chains. A scheme of the experimental set up is shown in Fig. 5.

The tip is fastened to a $z$ positioner which is moved by four stacks of shear piezos. To calibrate the displacement of the $z$ positioner, the light from a laser diode is focused into a single mode optical fiber and transmitted through a $2 \times 2$ directional coupler which splits the beam. Part of the light is coupled to a reference photodiode which measures the intensity of the laser beam. This intensity is the one used as reference when focusing the light. The remainder of the beam is transmitted to the end of the fiber which is placed close to a mirror glued to the rear of the $z$ positioner. In this way an interferometric cavity is formed between the fiber end and the mirror. About $95 \%$ of the beam that reaches the fiber end is transmitted, then reflected at the mirror and directed back into the fiber, interfering with the beam reflected at the fiber 


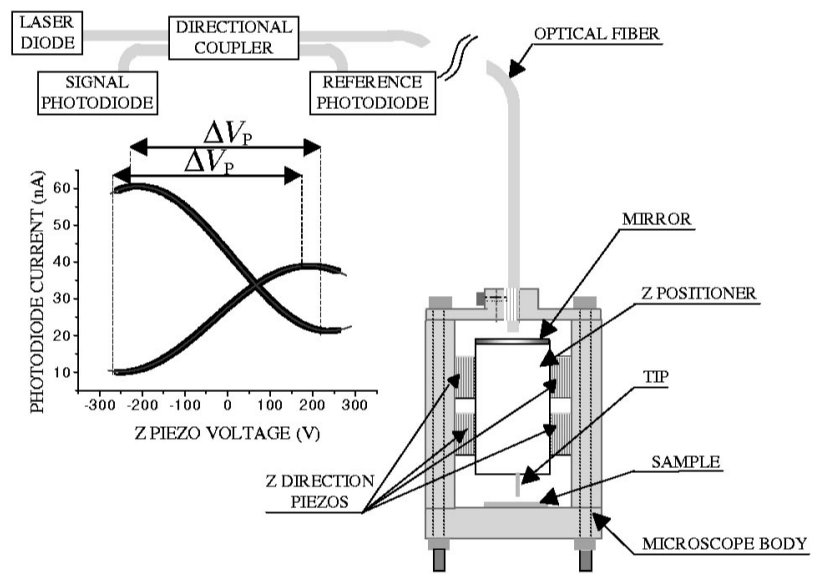

FIG. 5. Experimental setup for the STM configuration. The displacement of the tip against the sample is calibrated by an interferometric method which reliability and accuracy remain unaffected by the environment. The inset shows two different traces of the interference pattern measured with different initial interferometer cavity lengths.

end. The optical path difference between both beams - twice the interferometric cavity length-makes the intensity of the resulting beam to be given by

$$
I=A+B \cos (4 \pi d / \lambda+\delta),
$$

where $d$ is the interferometer cavity length, $A$ and $B$ are constants that decrease with $d$, and $\lambda$ is the wavelength of the laser beam, $660 \mathrm{~nm}$. The calibration is performed by approaching the $z$ positioner to the fiber until the intensity detected by the signal photodiode is sufficiently large. In order to vary linearly the cavity length, a voltage ramp is applied to the $z$ direction piezos while the photocurrent of the signal photodiode is measured. Two typical calibration measurements, with different initial interferometer cavity lengths (and therefore with different mean intensities) are shown in the graph in Fig. 5. For a voltage span $\Delta V_{p}$, the interference pattern traces a semiperiod. From Eq. (11), it follows that the ratio $\kappa$ between the displacement and the applied voltage is

$$
\kappa=\frac{\Delta d}{\Delta V_{p}}=\frac{\lambda}{4 \Delta V_{p}} .
$$

\section{EXPERIMENTAL RESULTS}

For the experiments we have used gold samples of better than $99.99 \%$ purity. For the STM experiments we have cleaned the sample with an $\mathrm{H}_{2} \mathrm{O}-\mathrm{H}_{2} \mathrm{SO}_{4}$ (1:3) solution and mechanically sharpened the tip, while for the MCBJ a fresh surface was formed at cryogenic vacuum when breaking the sample. The experiments were all performed at $4.2 \mathrm{~K}$. The conductance curves, from which the plateau lengths are obtained, are all measured at a constant bias voltage of $10 \mathrm{mV}$.

In Fig. 1 we show a typical experiment were an atomic chain is formed with the inset showing a histogram of lastplateau lengths. We have obtained length histograms with both STM and MCBJ.

A large number of indentation-elongation cycles of gold

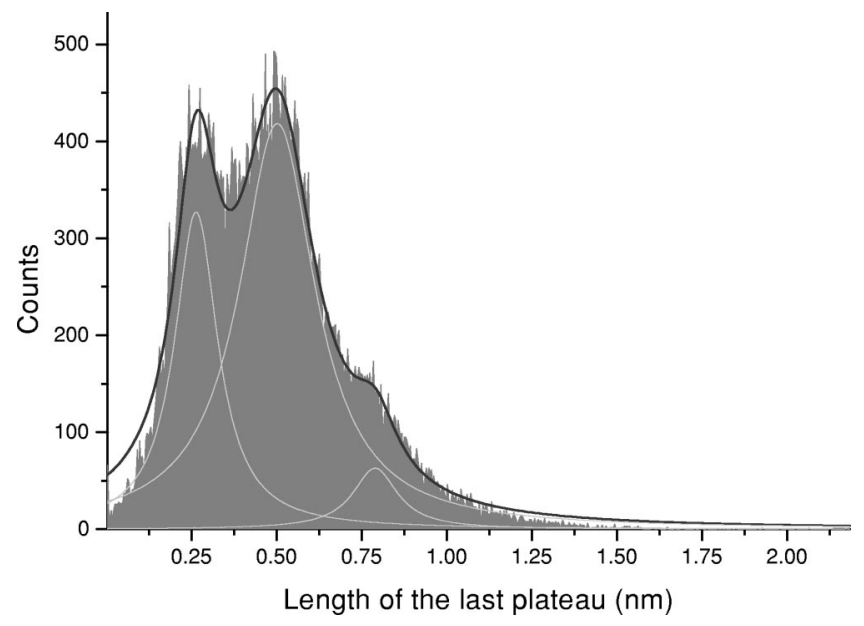

FIG. 6. Histogram of lengths for the last conductance plateau obtained in 65000 indentations made with the STM. We define the length of the last plateau as the distance between the point where the conductance drops below 1.2 times the conductance quantum and the one where the contact breaks.

nanocontacts was made. Special attention was given to include a large number of atomic configurations in the statistics, forcing structural rearrangements of a large number of atoms with frequent deep indentations of several hundreds of nanometers between cycles.

In the case of the MCBJ we have measured for several samples plateau length histograms and each of them was calibrated by both the tunnel barrier method and by means of the Gundlach oscillations. For the tunnel barrier method we have taken a work function for gold of $5.4 \mathrm{eV}$. In this case the standard deviation in the distribution of calibration values results into an error of $7 \%$. Using this calibration we obtain for the interpeak distance in the length histogram a value of $2.5 \pm 0.2 \AA$. The calibration using the Gundlach oscillation method was hampered most of the times by multiple tip effects in the field resonances and the response of the feedback to the applied voltage often had a very important quadratic term. Such complication appears to be characteristic for gold ${ }^{23}$ and the method works better for most other metals. As a result, we estimate the error in the calibration to be of the order of $20 \%$ and obtain for the interpeak distance $2.3 \pm 0.4 \AA$. We verified that the calibration obtained by the tunnel barrier method after admitting $\mathrm{He}$ gas into the chamber shifts the peak distance to $3.3 \AA$ in agreement with (Ref. 7).

In the case of the STM configuration, the calibration has been carried out by the interferometric method. This method has the advantage of being independent of tip and sample conditions. Using different lengths of the interferometer cavity, a value of $\kappa=3.70 \pm 0.13 \AA / \mathrm{V}$ is obtained. The experiments were all performed alternating the conductance with the calibration measurements every 15000 nanocontacts, while the instrument is maintained at $4.2 \mathrm{~K}$ in vacuum. In Fig. 6 we show the resultant length histogram from the STM measurements. We find here again a preference for contacts with one atom in cross section to break at specific values of length with a periodicity of $2.6 \pm 0.2 \AA$. 


\section{DISCUSSION}

The linear bond between two gold atoms is up to three times stronger than a bulk bond, as found in experiments and simulations. ${ }^{11,19,27}$ A single-atom gold contact can sustain a maximum tensile force of $1.5 \mathrm{nN}$ and before this limit is reached it is likely that atoms are pulled out of their position in the banks on either side of the contact. By repeating such atomic structural changes in the banks the one-atom contact evolves into a chain several atoms long. The chain finally breaks when the tensile force necessary to incorporate another atom from the nearby electrodes into the chain is higher than the breaking force of the chain itself. There will be characteristic interelectrode distances for which a chain of $n$ atoms is likely to break, as we will argue next.

Let us first discuss a length histogram for metals that do not easily form atomic chains, such as the $4 d$ metals $\mathrm{Rh}, \mathrm{Pd}$, and $\mathrm{Ag}$ investigated in Ref. 10. For these metals the length histogram shows only a single peak, usually at a shorter length than the first peak in the length histogram for $\mathrm{Au}$. We start counting the length of the plateau when the conductance drops to a typical value for a single atom contact, e.g., below $1.2 G_{0}$ for Ag. When pulling further the conductance remains roughly at this value while the bonds of the atom with the banks and those inside the banks are being stretched. As soon as the stored elastic energy reaches a maximum the contact breaks. The breaking point depends on the local atomic configurations in the banks near the contact and this leads to a certain width in the peak distribution. Thermal activation over the breaking barrier will also lead to a statistical distribution of observed values. The peak position in the length histogram shows the most probable length over which a one-atom contact can be stretched.

For chain-forming metals such as Au the first peak in the length histogram has the same interpretation as for those that break at a one-atom contact. Its position is at a longer length reflecting the stronger bond for low-coordination $\mathrm{Au}$ atoms. For all configurations giving rise to the distribution under the first peak there are equivalent configurations with the central atom replaced by two, three, or more atoms, forming a chain. These will give rise to additional peaks in the length histogram at multiples of the Au-Au bond distance in the chains, but stretched close to the breaking point. These distances are the ones at which the structure reaches the maximum tensile stress while it is not possible to introduce a new atom into the chain to relax it. If we consider that the force needed to break an atomic chain, $F_{b}$, is independent of the length of the chain $^{28}$ then the interelectrode distance at which the $n$-atoms chain breaks can be written as

$$
L_{n}=n L_{a t-a t}+(n+1) \frac{F_{b}}{K_{a}},
$$

where $L_{a t-a t}$ is the interatomic distance when no tension is applied and $K_{a}$ the elastic constant of the bond between atoms in the chain. Therefore the distance between the peaks in the plateau length histogram will be constant and equal to $\Delta=L_{a t-a t}+F_{b} / K_{a}$, or in other words, equal to the interatomic distance stretched to the point of breaking.
In this argumentation we have assumed that the banks are not shortened between the point at which the conductance first is seen to drop to the one-atom level and the final breaking point. As long as we limit the discussion to chains of only a few atoms in length this will be correct since the number of atomic layers in the banks will not be modified. Note that our value for the bond distance is based on the first two to four peaks and that atoms may fold in from both sides. Those events that result in a significant modification in the structure and effective length of the banks will only contribute to a smooth background in the length histogram. Only the chain-forming processes that conserve the structure of the banks are expected to be responsible for peaks at regular spacing in the length histogram, and these are thus expected to correspond to the atom-atom distance in the chains. The $\mathrm{Au}-\mathrm{Au}$ distance is measured from the distance between the peaks in the histogram, and we remark that the position of the first peak (relative to zero length) can differ from this value. For $\mathrm{Au}$ the first peak is nearly equal to the distance between the peaks, but different values have indeed been obtained, e.g., in the case of Pt chain length histograms. ${ }^{10}$

The bond distance near the anchoring points of the chain to the banks are expected to be about $10 \%$ shorter than the bond distance in the middle of the chain, as illustrated in the calculations by da Silva et al. ${ }^{16}$ A small variation in the bond length is consistent with our data, as can bee seen from the position of the fourth peak in Fig. 1. The fact that we derive our values for the bond distance mainly from the first three peaks implies that our result is biased toward the smaller distances at the anchoring points.

The interatomic distance for gold atomic chains quoted in Ref. 7 as $3.6 \AA \pm 30 \%$ was affected by calibration inaccuracies in two ways: a systematic error in the calibration due to He exchange gas condensed onto the gold surface, resulting in an overestimate of the $\mathrm{Au}-\mathrm{Au}$ distance. In addition, variations between various calibrations, which is reflected in the large uncertainty for the $\mathrm{Au}-\mathrm{Au}$ distance. We have now used three independent techniques for an improved calibration with an accuracy of approximately $7 \%$ for two of them, the interferometric method for the STM and the tunnel barrier method for the MCBJ, which show consistence between these techniques and gives a more accurate value of the bond distance in a chain formed by gold atoms. The calibration procedure with the largest error makes use of the Gundlach oscillations and gives a somewhat smaller, but consistent, value. However, there is still a problem of consistency with the results of $\mathrm{H}$. Ohnishi et al. ${ }^{8}$ and Rodrigues and Ugarte ${ }^{9}$ who report an interatomic distance of about 3.3-4.0 $\AA$ obtained from TEM images. The discrepancy could be due the different methods used to fabricate the chains or the difference in temperature and environmental conditions. It has been recently pointed out that there exists a possibility of incorporating one oxygen atom per gold atom in an atomic chain. $^{29}$ This addition should give rise to a gold-oxygen nanochain with a $\mathrm{Au}-\mathrm{Au}$ distance of $3.8 \AA$ for a relaxed chain and up to more than $4.2 \AA$ for a stretched one. This kind of chain should exhibit a conductance of one quantum unit. 


\section{CONCLUSIONS}

We have applied different calibration techniques for the MCBJ and STM in order to obtain a more accurate value for the distance between peaks in length histograms of the last plateau of conductance before rupture of gold contacts at low temperature. The values obtained for the interatomic distance in a chain of gold atoms at the point of breaking are 2.5 $\pm 0.2,2.3 \pm 0.4$, and $2.6 \pm 0.2 \AA$. We obtain an overall value for the interatomic distance of $2.5 \pm 0.2 \AA$, which closely agrees with results from model calculations.

\section{ACKNOWLEDGMENTS}

This work is part of the research program of the "Stichting FOM," which is financially supported by NWO, it was funded by the DGI under Contract No. MAT2001-1281 and the research has been supported by the Marie Curie Program of the European Community under Contract No. HPMF-CT2000-0072. R.G. acknowledges financial support from U.A.M. We thank O.I. Shklyarevskii and R.H.M. Smit for stimulating discussions.
${ }^{1}$ N. Agraï, J.G. Rodrigo, and S. Vieira, Phys. Rev. B 47, 12345 (1993).

${ }^{2}$ J.I. Pascual, J. Méndez, J. Gómez-Herrero, A.M. Baró, and N. García, Phys. Rev. Lett. 71, 1852 (1993).

${ }^{3}$ C.J. Muller, J.M. van Ruitenbeek, and L.J. de Jongh, Physica C 191, 485 (1992).

${ }^{4}$ N. Agraït, G. Rubio, and S. Vieira, Phys. Rev. Lett. 74, 3995 (1995).

${ }^{5}$ C. Untiedt, G. Rubio, S. Vieira, and N. Agraï, Phys. Rev. B 56, 2154 (1997).

${ }^{6}$ E. Scheer, N. Agraï, J.C. Cuevas, A. Levy Yeyati, B. Ludoph, A. Martín-Rodero, G. Rubio Bollinger, J.M. van Ruitenbeek, and C. Urbina, Nature (London) 394, 154 (1998); see also E. Scheer, P. Joyez, D. Esteve, C. Urbina, and H. Devoret, Phys. Rev. Lett. 78, 3535 (1997).

${ }^{7}$ A.I. Yanson, G. Rubio-Bollinger, H.E. van den Brom, N. Agraï, and J.M. van Ruitenbeek, Nature (London) 395, 783 (1998).

${ }^{8}$ H. Ohnishi, Y. Kondo, and K. Takayanagi, Nature (London) 395, 780 (1998).

${ }^{9}$ V. Rodrigues and D. Ugarte, Phys. Rev. B 63, 073405 (2000).

${ }^{10}$ R.H.M. Smit, C. Untiedt, A.I. Yanson, and J.M. van Ruitenbeek, Phys. Rev. Lett. 87, 266102 (2001).

${ }^{11}$ S. Bahn and K.W. Jacobsen, Phys. Rev. Lett. 87, 266101 (2001).

${ }^{12}$ M.R. Sørensen, M. Brandbyge, and K.W. Jacobsen, Phys. Rev. B 57, 3283 (1998).

${ }^{13}$ J.A. Torres, E. Tosatti, A. Dal Corso, F. Ecorcolessi, J.J. Kohanoff, T.D. Di Tolla, and J.M. Soler, Surf. Sci. 426, L441 (1999).

${ }^{14}$ D. Sánchez-Portal, E. Artacho, J. Junquera, P. Ordejón, A. García, and J.M. Soler, Phys. Rev. Lett. 83, 3884 (1999).

${ }^{15}$ H. Häkkinen, R.N. Barnett, A.G. Scherbakov, and U. Landman, J. Phys. Chem. B 104, 9063 (2000).

${ }^{16}$ E.Z. da Silva, A.J.R. da Silva, and A. Fazzio, Phys. Rev. Lett. 87, 256102 (2001).

${ }^{17}$ M. Okamoto and K. Takayanagi, Phys. Rev. B 60, 7808 (1999).

${ }^{18}$ L. De Maria and M. Springborg, Chem. Phys. Lett. 323, 293 (2000).

${ }^{19}$ G. Rubio, N. Agrait, and S. Vieira, Phys. Rev. Lett. 76, 2302 (1996).

${ }^{20}$ O.Yu. Kolesnychenko, O.I. Shklyarevskii, and H. van Kempen, Rev. Sci. Instrum. 70, 1442 (1999).

${ }^{21}$ J.G. Simmons, J. Appl. Phys. 34, 1793 (1963).

${ }^{22}$ G. Binnig, N. Garcia, H. Rohrer, J.M. Soler, and F. Flores, Phys. Rev. B 30, 486 (1984).

${ }^{23}$ O.Yu. Kolesnychenko, O.I. Shklyarevskii, and H. van Kempen, Phys. Rev. Lett. 83, 2242 (1999).

${ }^{24}$ H.B. Michaelson, J. Appl. Phys. 48, 4729 (1977).

${ }^{25}$ K.H. Gundlach, Solid-State Electron. 9, 946 (1966).

${ }^{26}$ T.R. Albrecht, P. Grütter, and D. Rugar, Ultramicroscopy 42-44, 1638 (1992).

${ }^{27}$ G. Rubio-Bollinger, S.R. Bahn, N. Agraï, K.W. Jacobsen, and S. Vieira, Phys. Rev. Lett. 87, 026101 (2001).

${ }^{28}$ It has been measured that this is the case at least for the small chains we consider here and that are no longer than seven atoms (Ref. 27).

${ }^{29}$ S. R. Bahn, Ph.D. thesis, Technical University of Denmark, September 2001. 